\title{
Cross-Sectional Study of Swallowing Phases in Cases of Megaesophagus Caused by Chagas Disease
}

\author{
Aretuza Zaupa Gasparim El Gharib ${ }^{\mathrm{a}}$, Roberto Oliveira Dantas , c $^{\mathrm{b}}$
}

\begin{abstract}
Background: Chagas disease, caused by the flagellate protozoan Trypanosoma cruzi, is an infectious cause of secondary achalasia and megaesophagus. Moreover, the oral and pharyngeal phases of swallowing may also be affected, which may contribute to dysphagia and increase the possibility of airway aspiration during and/or after swallowing. This cross-sectional study evaluated, with videofluoroscopy, the oral, pharyngeal, and esophageal phases of swallowing in patients with megaesophagus caused by Chagas disease. The hypothesis is that there is impairment of the pharyngeal phase of swallowing that may increase the risk of airway aspiration.
\end{abstract}

Methods: A total of 29 patients, aged 48 - 73 years (mean: $63.8 \pm 5.1$ years), with dysphagia, radiological changes in the esophagus, and positive serologic test for Chagas disease, participated in the study. They were submitted to the videofluoroscopic evaluation of oral, pharyngeal, and esophageal phases, swallowing twice $10 \mathrm{~mL}$ of liquid and $10 \mathrm{~mL}$ of thickened barium boluses.

Results: The most frequent findings were: oral residues and ineffective ejection in the oral phase; residues in vallecula, pharynx, and pyriform sinuses in the pharyngeal phase; abnormal esophageal motility, longer clearance, and longer transit in the esophageal phase. Laryngeal penetration was seen in $28 \%$ of the patients. Patients with increased esophageal diameter had more pharyngeal residues than patients without increased esophageal diameter. None of the patients had airway aspiration.

Conclusion: Megaesophagus caused by Chagas disease may affect all phases of swallowing, with an increase in oral and pharyngeal residues which suggest the impairment of oral and pharyngeal efficiency. None of the patients had airway aspiration.

Keywords: Chagas disease; Esophageal achalasia; Deglutition disorders; Esophageal diseases; Trypanosomiasis

Manuscript submitted August 13, 2021, accepted September 29, 2021

Published online October 14, 2021

aState University of Maringa, Maringa, Parana, Brazil

${ }^{b}$ Ribeirao Preto Medical School, University of Sao Paulo, Ribeirao Preto, Sao Paulo, Brazil

${ }^{\mathrm{c} C o r r e s p o n d i n g ~ A u t h o r: ~ R o b e r t o ~ O l i v e i r a ~ D a n t a s, ~ R i b e i r a o ~ P r e t o ~ M e d i c a l ~}$ School, University of Sao Paulo, Av. Bandeirantes, 3900, Campus da USP, Ribeirao Preto, SP, Brazil. Email: rodantas@fmrp.usp.br

doi: https://doi.org/10.14740/gr1458

\section{Introduction}

Chagas disease, a consequence of infection with flagellate protozoan Trypanosoma cruzi, affects mainly the heart and digestive system [1-5]. The whole digestive system has its function impaired to some degree $[4,5]$, whereas the changes caused by the disease in the esophagus and colon have clinical importance, as they can result in megaesophagus and megacolon, leading to dysphagia and constipation $[2,4,5]$.

The literature describes the sharp loss of neurons in the esophagus' myenteric plexus (Auerbach) [4-6], which is responsible for the esophagus smooth muscle motility control [7]. The striated muscles are present in the mouth, pharynx, and a small segment of the proximal esophagus, whose movements are controlled by the central nervous system [7-9].

Swallowing, which is extremely complex and dynamic, is the process with which the food is prepared, organized, and ejected from the oral cavity, goes through the pharynx and esophagus, and reaches the stomach $[8,9]$. Comprising a series of quick and integrated movements, swallowing has the function of transporting the food so that it nourishes and hydrates the person [9]. Therefore, it needs to be safe and effective in all its phases: oral, pharyngeal, and esophageal. It needs to be safe in terms of preventing swallowed material from entering the airways (which can happen in the pharyngeal phase and cause complications) and effective in terms of transporting all the swallowed material from the mouth to the stomach $[9,10]$.

The anatomical changes caused by Chagas disease take place in the esophagus, but the resulting esophagus motility abnormalities can cause changes in the oral and pharyngeal phases $[11,12]$ and impair both safety and effectiveness. The objectives of this investigation were to: 1) describe the changes in the oral, pharyngeal, and esophageal phases of swallowing found in the videofluoroscopy examination of patients with esophageal impairment due to Chagas disease; 2) compare patients with esophageal dilation with patients without esophageal dilation regarding residues, time of oral and pharyngeal transit, frequency of bolus airway penetration, and episodes of aspiration. The hypothesis is that patients whose esophagus has been impaired by the disease may have changes in all the phases of swallowing, with an increased risk of bolus airway aspiration, worse in patients with esophageal dilation.

\section{Materials and Methods}

All experiments were conducted in accordance with the Decla- 
ration of Helsinki. This research was approved by the Human Research Ethics Committee at the State University of Maringa (UEM), Brazil, with Certificate of Presentation for Ethical Consideration (CAAE) number 45350415.0.0000.0104, Institutional Review Board (IRB): 1.715.018.

The study was a cross-sectional investigation done in State University of Maringa (Brazil), Laboratory of Chagas Disease, in a convenience sample of consecutive patients of both sexes evaluated from December 2016 to May 2017, with a positive serologic test result for Chagas disease, radiological abnormalities in the esophagus, and complaints of dysphagia for more than a year. The exclusion criteria encompassed patients with a negative serologic test result for infection with Trypanosoma cruzi, with a history of neurological and/or oncological changes, previous treatment of Chagas esophagopathy, patients without dysphagia, and patients without esophageal abnormalities in radiological examination. The patients did not have malnutrition, dehydration or heart failure.

Chagasic esophagopathy was diagnosed with radiological examination of the esophagus, using abnormality criteria based on the megaesophagus classification described in 1960 $[13,14]$ and standard distances and volume of barium sulfate ingested, performed after $12 \mathrm{~h}$ of fasting. In this classification, the degree I refers to patients with slow transit and retention of contrast medium in the esophageal body, without an increase in the distal diameter. Degrees II, III, and IV refer to those who not only retain contrast medium in the esophageal body but also have an increased distal esophageal diameter - the greater the retention and diameter, the higher the degree, from II to IV. The limit to consider an increased distal diameter in the standard radiography was $4 \mathrm{~cm}$ [15].

For videofluoroscopy, the patients swallowed a liquid mixture of $25 \mathrm{~mL}$ of water at room temperature and $25 \mathrm{~mL}$ of liquid barium sulfate (Opti-Bar 66.7\%, Alko do Brasil, Taquara, RJ, Brazil). They also had a spoon-thick mixture, with $3.6 \mathrm{~g}$ of food thickener Resource ThickenUP Clear (Nestle Health Science, Osthofen, Germany) added to $50 \mathrm{~mL}$ of water at room temperature and $50 \mathrm{~mL}$ of liquid barium. The liquid was served in a $10 \mathrm{~mL}$ syringe and the thickened liquid, on a spoon, directly into the standing patients' mouth. They swallowed twice $10 \mathrm{~mL}$ of liquid bolus and twice $10 \mathrm{~mL}$ of thickened bolus, as prompted by the researcher. Esophageal phase was evaluated after the first swallowing of liquid bolus. The examination was conducted in the lateral position with a Toshiba radiological device (Japan), recording 30 frames per second. The liquid bolus was classified as level 0 (thin liquid) and the thickened bolus as level 3 (moderately thick) according to the International Dysphagia Diet Standardisation Initiative (IDDSI) [16].

The following parameters were observed in the videofluoroscopy: 1) In the oral phase of swallowing: change in labial function, change in tongue function, contrast residue in the tongue region, impaired bolus organization, inefficient food ejection, premature posterior spillage, and prolonged oral transit time (OTT), that is, lasting more than $1 \mathrm{~s}[12,17] .2)$ In the pharyngeal phase of swallowing: residues on the pharyngeal wall, residues in the vallecula, residues in the pyriform sinuses, prolonged pharyngeal transit time (PTT), laryngeal penetration, and airway aspiration. Laryngeal penetration occurred when the swallowed bolus entered the larynx but re- mained above the vocal folds; aspiration occurred when the swallowed material passed below the vocal folds. These were assessed with the Rosenbek penetration-aspiration scale [18]. Prolonged PTT occur when it last more than 1.5 s [17]. 3) In the esophageal phase: slow esophageal transit (i.e., lasting more than $10 \mathrm{~s}$ ) [19], residue in the upper esophageal sphincter, esophageal-pharyngeal reflux, change in esophageal motility, and difficult esophageal clearance.

The OTT, PTT, esophageal transit, and the quantification of residues were analyzed and calculated based on the images and video analysis, with the markers in the video editing program Kinovea - 0.8.15 (Copyright $^{\circledR}$ 2006-2011 - Joan Charmant and Contrib), which enables frame-by-frame analysis of the 30-frame-per-second record. The changes described were observed in at least one of the four swallows. The OTT was calculated after identifying the first frame with the movement of the bolus swallowed after prompted by the researcher until the first frame in which the tail part of the bolus reached the posterior part of the mandibular ramus. The PTT was measured from the frame in which the proximal (head) part of the bolus reached the posterior part of the mandibular ramus to the first frame in which the end of the bolus passed through the upper esophageal sphincter [17]. The residues were classified as follows: degree 0 - no residue; degree 1 - residue occupying less than $10 \%$ of the vallecula and pyriform sinuses; degree 2 - residues occupying more than $10 \%$ and less than $50 \%$ of the vallecula and pyriform sinuses [20]. The OTT, PTT, and the penetration-aspiration scale were compared between the patients whose distal esophageal diameter was not increased (degree $\mathrm{I}, \mathrm{n}=12$ ) and those whose distal esophageal diameter was increased (degree II, III, IV, $\mathrm{n}=17$ ). They were statistically analyzed with the Mann-Whitney test (for transit time) and Fisher exact test (for the residues and penetration-aspiration scale). Results with $\mathrm{P} \leq 0.05$ were considered significant.

\section{Results}

The study included 29 patients, $21(72 \%)$ women and eight (28\%) men, aged $46-73$ years (mean $63 \pm 5$ years). In the radiological examination, all of them retained contrast in the esophageal body: 12 without an increase in distal esophageal diameter (degree I) and 17 with an increase in distal diameter (nine in degree II, six in degree III, and two in degree IV). Twenty patients (69\%) had changed the consistency of the foods they ingested, from solid to pureed and/or liquid foods, as dysphagia progressed. Of the patients assessed, 97\% had changes in the oral phase, $93 \%$ in the pharyngeal phase, and $100 \%$ in the esophageal phase.

The most frequent changes in the oral phase of swallowing were the presence of oral residue in $97 \%$ of the patients, ineffective ejection in $72 \%$, and labial dysfunction in $55 \%$ (Table 1). Premature posterior bolus spillage was observed in $34 \%$ of the patients.

In the pharyngeal phase of swallowing, there were residues in the vallecula $(79 \%)$, pharynx $(79 \%)$, and pyriform sinuses $(41 \%)$. Bolus penetration into the larynx, without residues above the vocal folds, was observed in $28 \%$ of the patients 
Table 1. Results of the Videofluoroscopic Evaluation of the Oral Phase of Swallowing in Patients With Chagas Disease $(n=29)$

\begin{tabular}{lll} 
& Number & Percentage \\
\hline Prolonged oral transit & 2 & $7 \%$ \\
Change in labial function & 16 & $55 \%$ \\
Tongue dysfunction & 2 & $7 \%$ \\
Oral residue & 28 & $97 \%$ \\
Poorly organized bolus & 7 & $24 \%$ \\
Ineffective ejection & 21 & $72 \%$ \\
Premature posterior spillage & 10 & $34 \%$ \\
\hline
\end{tabular}

(Table 2). None of the patients had bolus airway aspiration. Degree 3 on the Rosenbek scale was observed, with the liquid bolus, in $16.7 \%$ of the swallows in patients without esophageal dilation and in $17.7 \%$ of the patients with dilation; and with the thickened bolus, in $8.3 \%$ of those without esophageal dilation and $11.8 \%$ of the patients with dilation $(\mathrm{P}=0.99)$.

In the esophagus, there was a long bolus clearance $(83 \%)$, impaired motility (72\%), and residues in the upper esophageal sphincter (72\%) (Table 3).

There was no difference $(\mathrm{P}>0.07)$ in OTT and PTT between patients with and without esophageal dilation (Table 4). Liquid bolus residues in the pharynx were more frequent in patients with an increased esophageal diameter (88\%) than in those without an increase $(55 \%)(\mathrm{P}=0.05$, Table 5).

\section{Discussion}

Assessments of oral and pharyngeal phases of swallowing in Chagas disease have been previously reported. The description includes long pharyngeal transit with a more consistent bolus [12], multiple swallows and reduced laryngeal elevation [21], residues in the pharynx and laryngeal penetration [22], and a similar sequence of the events associated with swallowing [23]. Studies with scintigraphy have described a longer time to clear the pharynx and for the bolus to pass from the pharynx to the esophagus $[24,25]$. These papers reported no occurrences of aspiration.

In Chagas disease, the esophageal motor changes have been known for a long time, the similar changes described for idiopathic achalasia $[4,5,26,27]$. They are a consequence of loss in the myenteric plexus [4-6], located between the circular and longitudinal muscle layers, mainly responsible for motility

Table 2. Results of the Videofluoroscopic Evaluation of the Pharyngeal Phase of Swallowing in Patients With Chagas Disease $(n=29)$

\begin{tabular}{lll} 
& Number & Percentage \\
\hline Residues in the vallecula & 23 & $79 \%$ \\
Residues in the pharynx & 23 & $79 \%$ \\
Residues in pyriform sinuses & 12 & $41 \%$ \\
Laryngeal penetration & 8 & $28 \%$ \\
\hline
\end{tabular}

Table 3. Results of the Videofluoroscopic Evaluation of the Esophageal Phase of Swallowing in Patients With Chagas Disease $(n=29)$

\begin{tabular}{lll}
\hline & Number & Percentage \\
\hline Residues in upper sphincter & 21 & $72 \%$ \\
Esophageal-pharyngeal reflux & 3 & $10 \%$ \\
Abnormal esophageal motility & 21 & $72 \%$ \\
Long esophageal clearance & 24 & $83 \%$ \\
\hline
\end{tabular}

control in the esophageal segment with smooth muscles [8]. The proximal part, with striated muscles, is controlled by the central nervous system [7].

The different phases of swallowing are mutually independent. However, what takes place in one of them can influence the others [7], and they depend on the feedback of what happens in the pharynx and esophagus. In Chagas disease, the central nervous system is not significantly changed [28, 29]; therefore, functional changes in the esophageal phase of swallowing may influence the oral and pharyngeal phases, with adaptations aimed at ensuring safety and effectiveness in swallowing.

There is an important impairment of the esophageal phase of swallowing caused by Chagas disease, with difficult bolus transit, bolus retention in the esophagus, dilation of the organ, and regurgitation of the material retained $[4,5,30]$. This videofluoroscopy assessment particularly revealed abnormal esophageal motility and difficulty for the swallowed material to pass to the stomach.

The most frequent changes observed in the oral phase were the presence of oral residue, ineffective oral ejection, and premature posterior spillage; and in the pharyngeal phase, there were residues and laryngeal penetration. These changes occur in chagasic patients more than expected for normal people [31]. Such changes in the oral and pharyngeal phases of swallowing may contribute to these patients' dysphagia. However, the significant change in esophageal motility must be the main cause of dysphagia, while the other changes are secondary to the megaesophagus.

The changes observed in the pharyngeal phase of swallowing may be a consequence of megaesophagus, which is sug-

Table 4. OTT and PTT (in Seconds) of $10 \mathrm{~mL}$ of Liquid and Thickened Boluses in Patients With Chagas Disease Without an Increase in Distal Esophageal Diameter $(n=12)$ and With an Increase in Distal Esophageal Diameter $(n=17)$

\begin{tabular}{|c|c|c|c|}
\hline & Without increase & With increase & $\mathbf{P}$ \\
\hline \multicolumn{4}{|l|}{ OTT } \\
\hline Liquid & $0.51(0.32-0.63)$ & $0.68(0.45-0.90)$ & 0.08 \\
\hline Thickened & $0.70(0.50-1.38)$ & $0.84(0.57-1.43)$ & 0.53 \\
\hline \multicolumn{4}{|l|}{ PTT } \\
\hline Liquid & $0.24(0.17-0.37)$ & $0.33(0.26-0.40)$ & 0.24 \\
\hline Thickened & $0.24(0.20-0.45)$ & $0.30(0.23-0.60)$ & 0.32 \\
\hline
\end{tabular}

Results in median and interquartile values. OTT: oral transit time; PTT: pharyngeal transit time. 
Table 5. Scale of Residues in the Vallecula, Pharynx, and/or Pyriform Sinuses in Patients With Chagas Disease Without an Increase in Distal Esophageal Diameter $(n=12)$ and Patients With an Increase in Distal Esophageal Diameter $(n=17)$ After Swallowing 10 $\mathrm{mL}$ of Liquid and Thickened Boluses

\begin{tabular}{|c|c|c|c|c|c|}
\hline \multirow{2}{*}{ Scale } & \multicolumn{2}{|c|}{ Without increase } & \multicolumn{2}{|c|}{ With increase } & \multirow{2}{*}{$\mathbf{P}$} \\
\hline & Number & Percentage & Number & Percentage & \\
\hline Liquid & & & & & 0.05 \\
\hline 1 & 5 & $42 \%$ & 14 & $82 \%$ & \\
\hline 2 & 1 & $8 \%$ & 1 & $6 \%$ & \\
\hline 0 & 7 & $58 \%$ & 4 & $23 \%$ & \\
\hline 1 & 5 & $42 \%$ & 12 & $71 \%$ & \\
\hline 2 & 0 & $0 \%$ & 1 & $6 \%$ & \\
\hline
\end{tabular}

gested by the results of assessments made in idiopathic achalasia. This disease has similar pathophysiology and treatment [32] to that of chagasic achalasia, also causing megaesophagus, describing pharyngeal contraction asymmetry, residues, and diverticula on the lateral wall [33], hypertensive upper esophageal sphincter [34], and effortful pharyngeal swallows [35].

The objective of decreasing the consistency of foods ingested is to make them easier to pass through the esophagus. However, the occurrence of residues in the pharyngeal phase, especially in the vallecula [36], associated with a decreased food consistency, increased the risk of airway aspiration [36-38]. The videofluoroscopic results point to changes that increase the possibility of aspiration, namely, in the oral phase - reduced lip closure, residue in the oral cavity, prolonged oral transit; in the pharyngeal phase - delayed onset, reduced laryngeal elevation, residues in the vallecula, pyriform sinuses, and on the pharyngeal wall, and prolonged transit [20]. This investigation found $79 \%$ of the patients with residues in the vallecula and pharynx (impaired efficiency) and $28 \%$ with laryngeal penetration (impaired safety). The larynx does not elevate properly [21, 22], and there is a higher pressure on the upper esophageal sphincter and slow pharyngeal transit [11], which can cause food to be retained in the pharynx.

However, none of the patients investigated had a history of frequent pneumonia, although airway aspiration did not always cause pneumonia, and no aspiration was observed during or after swallowing in any of the patients. No previous papers have made any reference to aspiration associated with swallowing occurring in patients with idiopathic and chagasic achalasia. This indicates that the airway protective mechanisms may be working properly.

The long experience with Chagas disease patients in countries in Latin America and other continents $[1,2]$ shows no evidence that they have more pulmonary infections than the population in general. However, cases with esophageal dilation and content retention are exposed to aspiration of such esophageal content, which may happen after esophageal-pharyngeal reflux [39].

This investigation has limitations. More patients could have been included, although those who were studied demon- strated the changes observed in chagasic esophagopathy and were enough to reach conclusions. There were a greater number of women assessed, which may have influenced the results - although this is still an unconfirmed possibility. Esophageal manometric examination was not performed; however, this examination is not essential to demonstrate the esophageal involvement by Chagas disease [14].

\section{Conclusion}

Patients with esophagopathy due to Chagas disease may have changes in the oral and pharyngeal phases of swallowing. Residues in the mouth and pharynx are often observed; however, no airway aspiration was found.

\section{Acknowledgments}

None to declare.

\section{Financial Disclosure}

This investigation did not receive any specific grant from funding agencies.

\section{Conflict of Interest}

The authors Aretuza ZG EL Gharib and Roberto O Dantas declare that there is no conflict of interest related to this manuscript. Dr Roberto O Dantas is a member of the Board of Directors of the International Dysphagia Diet Standardisation Initiative (IDDSI).

\section{Informed Consent}

All participants provided written informed consent. 


\section{Author Contributions}

Aretuza ZG El Gharib collected the data. Aretuza ZG El Ghar$\mathrm{ib}$ and Roberto O Dantas designed the study, analyzed and interpreted the data, wrote the manuscript and made the decision to submit to publication.

\section{Data Availability}

The authors declare that data supporting the findings of this study are available within the article.

\section{References}

1. Lidani KCF, Andrade FA, Bavia L, Damasceno FS, Beltrame MH, Messias-Reason IJ, Sandri TL. Chagas disease: from discovery to a worldwide health problem. Front Public Health. 2019;7:166.

2. Zheng C, Quintero O, Revere EK, Oey MB, Espinoza F, Puius YA, Ramirez-Baron D, et al. Chagas disease in the New York City metropolitan area. Open Forum Infect Dis. 2020;7(5):ofaa156.

3. Marin-Neto JA, Cunha-Neto E, Maciel BC, Simoes MV. Pathogenesis of chronic Chagas heart disease. Circulation. 2007;115(9):1109-1123.

4. de Oliveira RB, Troncon LE, Dantas RO, Menghelli UG. Gastrointestinal manifestations of Chagas' disease. Am J Gastroenterol. 1998;93(6):884-889.

5. Matsuda NM, Miller SM, Evora PR. The chronic gastrointestinal manifestations of Chagas disease. Clinics (Sao Paulo). 2009;64(12):1219-1224.

6. Koberle F. Chagas' disease and Chagas' syndromes: the pathology of American trypanosomiasis. Adv Parasitol. 1968;6:63-116.

7. Lang IM. Brain stem control of the phases of swallowing. Dysphagia. 2009;24(3):333-348.

8. Shaw SM, Martino R. The normal swallow: muscular and neurophysiological control. Otolaryngol Clin North Am. 2013;46(6):937-956.

9. Sasegbon A, Hamdy S. The anatomy and physiology of normal and abnormal swallowing in oropharyngeal dysphagia. Neurogastroenterol Motil. 2017;29(11):e13100.

10. Matsuo K, Palmer JB. Anatomy and physiology of feeding and swallowing: normal and abnormal. Phys Med Rehabil Clin N Am. 2008;19(4):691-707, vii.

11. Devkota S. The gut microbiome during acute lifestyle transition. Nat Med. 2020;26(7):1013-1015.

12. dos Santos CM, Cassiani RA, Dantas RO. Videofluoroscopic evaluation of swallowing in Chagas' disease. Dysphagia. 2011;26(4):361-365.

13. de RJ, Lauar KM, de OA. [Clinical and radiological aspects of aperistalsis of the esophagus]. Rev Bras Gastroenterol. 1960;12:247-262.

14. Abud TG, Abud LG, Vilar VS, Szejnfeld D, Reibscheid S. Radiological findings in megaesophagus secondary to Chagas disease: chest X-ray and esophagogram. Radiol
Bras. 2016;49(6):358-362.

15. Dantas RO, Alves LMT, Cassiani RA, Santos CM. Clinical measurement of swallowing and proximal esophageal contractions in Chagas' disease. Esophagus. 2009;6(4):231-236.

16. Cichero JAY, Lam PTL, Chen J, Dantas RO, Duivestein J, Hanson B, Kayashita J, et al. Release of updated International Dysphagia Diet Standardisation Initiative Framework (IDDSI 2.0). J Texture Stud. 2020;51(1):195-196.

17. Daniels SK, Schroeder MF, McClain M, Corey DM, Rosenbek JC, Foundas AL. Dysphagia in stroke: Development of a standard method to examine swallowing recovery. J Rehabil Res Dev. 2006;43(3):347-356.

18. Rosenbek JC, Robbins JA, Roecker EB, Coyle JL, Wood JL. A penetration-aspiration scale. Dysphagia. 1996;11(2):93-98.

19. Sand A, Ham H, Piepsz A. Oesophageal transit patterns in healthy subjects. Nucl Med Commun. 1986;7(10):741745.

20. Han TR, Paik NJ, Park JW. Quantifying swallowing function after stroke: A functional dysphagia scale based on videofluoroscopic studies. Arch Phys Med Rehabil. 2001;82(5):677-682.

21. dos Santos CM, Cassiani RA, Dantas RO. Clinical evaluation of swallowing in Chagas' disease. Rev Soc Bras Fono. 2011;16:215-220.

22. Cabral DMG, Abrahao Junior LJ, Marques CHD, Pereira BB, Pedrosa RC. Oropharyngeal dysphagia in patients with chronic Chagas disease: phonoaudiological, videofluoroscopic, and manometric evaluations. Acta Fisiatr. 2015;22(1):24-29.

23. dos Santos CM, Cassiani RA, do Nascimento WV, Dantas RO. Timing of pharyngeal swallow events in Chagas' disease. Gastroenterology Res. 2014;7(3-4):93-97.

24. Gomes FR, Secaf M, Kubo TT, Dantas RO. Oral and pharyngeal transit of a paste bolus in Chagas' disease. Dysphagia. 2008;23(1):82-87.

25. Souza MAN, Dantas RO, De Oliveira RB, Braga FJ. A scintigraphic study of oropharyngeal swallowing dynamics in Chagas' disease. Neurogastroenterol Motil. 2000;12(4):335-341.

26. Rieder E, Fernandez-Becker NQ, Sarosiek J, Guillaume A, Azagury DE, Clarke JO. Achalasia: physiology and diagnosis. Ann N Y Acad Sci. 2020;1482(1):85-94.

27. Rogers AB, Rogers BD, Gyawali CP. Pathophysiology of achalasia. Ann Esophagus. 2020;3:27.

28. Pittella JE. Central nervous system involvement in Chagas disease: a hundred-year-old history. Trans R Soc Trop Med Hyg. 2009;103(10):973-978.

29. Wackermann PV, Fernandes RM, Elias J, Jr., Dos Santos AC, Marques W, Jr., Barreira AA. Involvement of the central nervous system in the chronic form of Chagas' disease. J Neurol Sci. 2008;269(1-2):152-157.

30. de Oliveira RB, Rezende Filho J, Dantas RO, Iazigi N. The spectrum of esophageal motor disorders in Chagas' disease. Am J Gastroenterol. 1995;90(7):1119-1124.

31. Steele CM, Peladeau-Pigeon M, Barbon CAE, Guida BT, Namasivayam-MacDonald AM, Nascimento WV, Smaoui S, et al. Reference values for healthy swallow- 
ing across the range from thin to extremely thick liquids. J Speech Lang Hear Res. 2019;62(5):1338-1363.

32. Dantas RO. Management of esophageal dysphagia in chagas disease. Dysphagia. 2021;36(3):517-522.

33. Jones B, Donner MW, Rubesin SE, Ravich WJ, Hendrix TR. Pharyngeal findings in 21 patients with achalasia of the esophagus. Dysphagia. 1987;2(2):87-92.

34. Chavez YH, Ciarleglio MM, Clarke JO, Nandwani M, Stein E, Roland BC. Upper esophageal sphincter abnormalities: frequent finding on high-resolution esophageal manometry and associated with poorer treatment response in achalasia. J Clin Gastroenterol. 2015;49(1):17-23.

35. Menezes MA, Herbella FA, Patti MG. High-Resolution manometry evaluation of the pharynx and upper esophageal sphincter motility in patients with achalasia. J Gastrointest Surg. 2015;19(10):1753-1757.
36. Molfenter SM, Steele CM. The relationship between residue and aspiration on the subsequent swallow: an application of the normalized residue ratio scale. Dysphagia. 2013;28(4):494-500.

37. Steele CM, Peladeau-Pigeon M, Barrett E, Wolkin TS. The risk of penetration-aspiration related to residue in the pharynx. Am J Speech Lang Pathol. 2020;29(3):16081617.

38. Seo ZW, Min JH, Huh S, Shin YI, Ko HY, Ko SH. Prevalence and severity of dysphagia using videofluoroscopic swallowing study in patients with aspiration pneumonia. Lung. 2021;199(1):55-61.

39. Alves LR, Soares EG, Aprile LR, Elias-Junior J, Vilas Boas PP, Baddini-Martinez J. Chlorophyllin-stained macrophages as markers of pulmonary aspiration. Am J Respir Crit Care Med. 2013;188(12):1470-1472. 